\title{
Lactobacillus crispatus BC5 Interferes With Chlamydia trachomatis Infectivity Through Integrin Modulation in Cervical Cells
}

\section{OPEN ACCESS}

Edited by:

Alain Pierre Gobert,

Vanderbilt University Medical Center,

United States

Reviewed by:

Bruno Pozzetto,

Université Jean Monnet, France

Amanda J. Brinkworth,

Washington State University,

United States

Alan Paul Hudson,

Wayne State University School

of Medicine, United States

Alison Favaroni,

Friedrich-Loeffler-Institut, Germany

*Correspondence:

Beatrice Vitali

b.vitali@unibo.it

Antonella Marangoni

antonella.marangoni@unibo.it

${ }^{\dagger}$ These authors have contributed equally to this work

Specialty section:

This article was submitted to

Infectious Diseases,

a section of the journal

Frontiers in Microbiology

Received: 30 July 2018 Accepted: 16 October 2018 Published: 06 November 2018

Citation:

Parolin C, Frisco G, Foschi C,

Giordani B, Salvo M, Vitali B,

Marangoni A and Calonghi N (2018)

Lactobacillus crispatus BC5 Interferes With Chlamydia trachomatis Infectivity

Through Integrin Modulation

in Cervical Cells.

Front. Microbiol. 9:2630.

doi: 10.3389/fmicb.2018.02630

\section{Carola Parolin 1t, Giulia Frisco'1t, Claudio Foschi2t, Barbara Giordani', Melissa Salvo², Beatrice Vitali ${ }^{*}$, Antonella Marangoni ${ }^{2 *}$ and Natalia Calonghi ${ }^{1}$}

${ }^{1}$ Department of Pharmacy and Biotechnology, University of Bologna, Bologna, Italy, ${ }^{2}$ Microbiology, Department of Experimental, Diagnostic and Specialty Medicine, University of Bologna, Bologna, Italy

Lactobacilli play a crucial role in maintaining the ecological equilibrium of the vaginal niche, preventing the colonization of exogenous microorganisms. Although many studies have discussed the mechanisms displayed by lactobacilli in counteracting several urogenital pathogens, a few data are available on the interaction between lactobacilli and Chlamydia trachomatis. This study aimed to elucidate the molecular bases of the interaction among vaginal lactobacilli, the sexually transmitted pathogen C. trachomatis and the epithelial cervical cells. We evaluated the in vitro activity of 15 Lactobacillus strains, belonging to different species (i.e., L. crispatus, L. gasseri, L. vaginalis), against $C$. trachomatis. In particular, we evaluated the capability of lactobacilli cells to interfere with $C$. trachomatis infection in HeLa cells, by exclusion assays. Lactobacilli significantly reduced $C$. trachomatis infectivity, being $L$. crispatus the most active species. Although a dose-dependent effect was noticed, a significant antagonistic activity was maintained even at lower doses. As other Gram-positive bacteria (i.e., Streptococcus agalactiae, Enterococcus faecalis, and Bacillus subtilis) failed to interfere with $C$. trachomatis infectivity, Lactobacillus activity proved to be specific. The potential mechanism of protection was investigated in Lactobacillus crispatus BC5, chosen as the model strain. The incubation of HeLa cell line with BC5 cells induced important modifications in the epithelial plasma membrane, by altering lipid composition and $\alpha 5$ integrin subunit exposure. When $\alpha 5$ integrin subunits were masked by a specific blocking antibody or ITGA5 gene expression was silenced, Chlamydia infection was significantly reduced. It follows that $\alpha 5$ integrin subunit is crucial for the pathogen infection process, and the anti-Chlamydia activity can be directly linked to membrane properties modifications in cervical cells. The three Grampositive bacteria used as controls failed to modify the expression of $\alpha 5 \beta 1$ integrin. In conclusion, we identified a potential molecular mechanism at the basis of the protection exerted by $L$. crispatus BC5 against $C$. trachomatis, getting insights into the role of the cervico-vaginal microbiota for the woman's health.

Keywords: Chlamydia trachomatis, lactobacilli, HeLa cells, integrin, women health, probiotics, STIs 


\section{INTRODUCTION}

Chlamydia trachomatis (CT) represents the agent of the most common bacterial sexually transmitted infection (STI) worldwide (ECDC, 2015). In women, urogenital CT infections are often asymptomatic, thus remaining unnoticed and untreated. This can lead to complications and sequelae including pelvic inflammatory disease, tubal infertility, and ectopic pregnancy (Price et al., 2013; Menon et al., 2015).

A normal vaginal microbiota, dominated by lactobacilli, is crucial for the prevention of several urogenital and sexually transmitted infections, including Chlamydia (Gupta et al., 1998; Spurbeck and Arvidson, 2008; Parolin et al., 2015; Nardini et al., 2016; Foschi et al., 2017; Ñahui Palomino et al., 2017). This aspect is strengthened by the demonstration that in case of bacterial vaginosis, a clinical condition characterized by the depletion of lactobacilli, a higher risk of STI transmission and acquisition is reported (Taha et al., 1998; Martin et al., 1999; Wiesenfeld et al., 2003; Abbai et al., 2015).

The protective role of lactobacilli against urogenital pathogens is exerted through different mechanisms including the production of various antibacterial compounds (lactic acid, hydrogen peroxide, bacteriocins, and biosurfactants), the competitive exclusion for epithelial adhesion and the immunomodulation (Kaewsrichan et al., 2006; Borges et al., 2014; Parolin et al., 2015; Younes et al., 2018). In this context, the use of probiotic lactobacilli for the prevention and treatment of several urinary and vaginal tract infections has been extensively evaluated, with different results depending on the Lactobacillus species, the strain origin, the concentrations used and the outcome considered (Barrons and Tassone, 2008; Bolton et al., 2008; Spurbeck and Arvidson, 2011; Vitali et al., 2016).

Until now, only a few studies have focused on the in vitro interaction between lactobacilli and CT and many aspects remain to be elucidated (Gong et al., 2014; Mastromarino et al., 2014; Nardini et al., 2016). Considering that CT is an obligate intracellular bacterium, characterized by a unique biphasic developmental cycle alternating between the extracellular infectious 'elementary body' (EB) and the intracellular 'reticulate body' (RB) (Moulder, 1991), lactobacilli can interfere with CT infectivity acting on the different steps of its cycle.

Previous studies shed light on the metabolic interaction between $\mathrm{CT}$ and lactobacilli, mimicking what happens in the acid environment of the vaginal niche (Gong et al., 2014; Nardini et al., 2016), but they did not evaluate the ability of lactobacilli cells to compete and interfere with CT EBs infectivity in epithelial cells. It has also been reported that the interaction of lactobacilli with cervical cells results in changes in the structure/functions of the plasma membrane of epithelial cells, especially at the level of $\alpha 5 \beta 1$ integrin exposure (Calonghi et al., 2017). The integrin family of receptors is a major target for bacterial pathogens that colonize human tissues or invade specific cell types (Hoffmann et al., 2011; Hauck et al., 2012). Integrins are heterodimeric transmembrane receptors that mediate cell-cell and cell-extracellular matrix adhesion and, as a result, regulate many aspects of cell behavior. In addition to providing a physical transmembrane link between the extracellular environment and the cytoskeleton, they are capable of transducing bi-directional signals across the cell membrane (Hynes, 2002). In this context, the interaction of chlamydial Ctad1 adhesin with $\beta 1$ integrin subunit has been proposed as one mechanism for EBs binding, invasion, and signaling during entry into host epithelial cells (Elwell et al., 2016; Stallmann and Hegemann, 2016).

The aim of this study was to identify vaginal Lactobacillus strains capable of interfering with the infectious process of CT in cervical cells (HeLa cell line) and to understand the rationale of this interaction. A L. crispatus strain was chosen as a model to study the molecular mechanisms underlying the anti-Chlamydia activity, with particular reference to the modulation of plasma membrane properties and integrin role in HeLa cell line.

\section{MATERIALS AND METHODS}

\section{Bacterial Strains and Culture Conditions}

All the 15 Lactobacillus strains included in this study (Figure 1) were previously isolated from vaginal swabs of healthy premenopausal Caucasian women (Parolin et al., 2015). Lactobacilli were grown in de Man, Rogosa and Sharpe (MRS) broth supplemented with $0.05 \% \mathrm{~L}$-cysteine, for $18 \mathrm{~h}$ at $37^{\circ} \mathrm{C}$, in anaerobic atmosphere. Anaerobic conditions were achieved by using anaerobic jars supplemented with GazPack EZ (Becton Dickinson and Company, Sparks, MD, United States).

As specificity control, clinical isolates of Streptococcus agalactiae and Enterococcus faecalis were used. These strains were part of a broad collection including bacteria isolated from vaginal swabs submitted to the Microbiology Laboratory of Sant'OrsolaMalpighi University Hospital of Bologna (Italy) for routine diagnostic procedures. These two strains were grown in Brain Heart Infusion (BHI) broth, in $5 \% \mathrm{CO}_{2}$ for $18 \mathrm{~h}$ at $37^{\circ} \mathrm{C}$. Bacillus subtilis ATCC 31324 was also included as rod-shaped bacterium control. It was grown aerobically in BHI broth for $18 \mathrm{~h}$ at $37^{\circ} \mathrm{C}$. All culture media were purchased from Becton Dickinson and Company.

Bacterial cultures were centrifuged at $5,000 \times g$ for $10 \mathrm{~min}$ at $4^{\circ} \mathrm{C}$. Cell pellets were washed and re-suspended in sterile saline to obtain stock cell suspensions of $5 \times 10^{8} \mathrm{CFU} / \mathrm{mL}$.

\section{C. trachomatis Propagation and Purification of EBs}

In this study, CT strain $\mathrm{GO} / 86$, serotype $\mathrm{D}$, was used (Marangoni et al., 2015; Nardini et al., 2016). This strain was clinically isolated from a urethral swab of a patient with non-gonococcal urethritis, submitted to the Microbiology Laboratory of Sant'OrsolaMalpighi Hospital of Bologna, for routine diagnostic procedures and belongs to the laboratory collection. HeLa cells (ATCC CCL-2), a cell line originated from a human cervix carcinoma, were grown to confluent monolayers in $5 \% \mathrm{CO}_{2}$ at $37^{\circ} \mathrm{C}$, inside a Forma Series II 3110 Water-Jacketed $\mathrm{CO}_{2}$ Incubator (Thermo Fisher Scientific, Waltham, MA, United States). CT was propagated in HeLa cells, cultured in Dulbecco's minimal essential medium (DMEM) (EuroClone, Pero, Italy), supplemented with $10 \%$ fetal bovine serum, $1 \%$ L-glutamine $200 \mathrm{mM}$, and antibiotics (vancomycin $10 \mathrm{mg} / \mathrm{L}$, gentamicin 
$10 \mathrm{mg} / \mathrm{L}$, and amphotericin B $0.3 \mathrm{mg} / \mathrm{L}$ ). For the preparation of EBs, confluent HeLa cells were infected with CT in DMEM medium supplemented with cycloheximide $1 \mu \mathrm{g} / \mathrm{mL}$, centrifuged at $640 \times g$ for $2 \mathrm{~h}$ to facilitate cell penetration, then incubated at $37^{\circ} \mathrm{C}$ with $5 \% \mathrm{CO}_{2}$ for $48 \mathrm{~h}$ (Mastromarino et al., 2014). HeLa cells were then detached and fragmented by sonication, by using a Bandelin sonicator at minimum power. Samples were centrifuged at $500 \times \mathrm{g}$ for $10 \mathrm{~min}$ at $4^{\circ} \mathrm{C}$, and supernatants, which contain EBs, were further centrifuged at $40,000 \times g$ at $4^{\circ} \mathrm{C}$ for $1 \mathrm{~h}$. The resulting pellets, containing the purified $\mathrm{EBs}$, were re-suspended in sucrose-phosphate-glutamate (SPG) $0.2 \mathrm{M}$, divided into small aliquots and stored at $-80^{\circ} \mathrm{C}$.

\section{C. trachomatis Infectivity Interference Assay}

To study the capacity of lactobacilli cells to interfere with the entry of CT EBs into HeLa cells, exclusion experiments were performed (Parolin et al., 2015). HeLa cells were seeded at $2 \times 10^{4}$ cells $/ \mathrm{cm}^{2}$ in individual tubes containing sterile glass coverslips in $1 \mathrm{~mL}$ of medium, and allowed to reach $95-100 \%$ of confluence (approximately $1.25 \times 10^{5}$ cells). Lactobacillus cells $(0.1 \mathrm{~mL}$ of the stock cell suspension, corresponding to $5 \times 10^{7} \mathrm{CFU}$ ) were incubated for $1 \mathrm{~h}$ at $37^{\circ} \mathrm{C}$ with $5 \% \mathrm{CO}_{2}$ on HeLa cells. Afterward, $5 \times 10^{3}$ CT EBs $\left(0.01 \mathrm{~mL}\right.$ of a stock solution of $\left.5 \times 10^{5} \mathrm{EBs} / \mathrm{mL}\right)$ were added and further incubated for $1 \mathrm{~h}$ [multiplicity of infection $(\mathrm{MOI})=0.04]$. HeLa tubes infected with $5 \times 10^{3} \mathrm{CT}$ EBs without lactobacilli were used as controls. All the experiments were conducted in triplicate. At the end of the incubation, each HeLa tube was first washed three times with phosphate buffer saline (PBS), then $1 \mathrm{~mL}$ of complete medium, supplemented with $1 \mu \mathrm{g} / \mathrm{mL}$ cycloheximide, was added. Individual tubes were centrifuged at $640 \times g$ for $2 \mathrm{~h}$ to facilitate cell penetration and then incubated at $37^{\circ} \mathrm{C}$ with $5 \% \quad \mathrm{CO}_{2}$ for $48 \mathrm{~h}$. CT infection was evaluated by counting chlamydia inclusion forming units (IFUs) by direct immunofluorescence, using a monoclonal antibody against the chlamydial membrane lipopolysaccharide antigen conjugated with fluorescein (Meridian, Cincinnati, $\mathrm{OH}$, United States), as previously reported (Marangoni et al., 2015). Slides were observed under epi-fluorescence microscope (Eclipse E600, Nikon, Tokyo, Japan) equipped with a super high pressure mercury lamp and Plan Fluor DLL 20, 40, 100× lenses. The number of IFUs was counted in 30 randomly chosen $200 \times$ microscopic fields. Results were expressed as the percentage (median percentage \pm median absolute deviation) of CT infectivity, comparing the number of IFUs of the single experiments with the control tubes.

In order to exclude a potential effect of an acidic environment on CT EBs infectivity, the $\mathrm{pH}$ values of the culture medium were measured before the addition of lactobacilli cells and at the end of the incubation.

Exclusion experiments were also performed with $S$. agalactiae, E. faecalis, and B. subtilis cells instead of lactobacilli, applying the same conditions described above, to assess the specificity of the anti-CT activity.

Lactobacillus crispatus BC4, L. crispatus BC5 and L. gasseri BC14, selected among the most active strains in counteracting chlamydial infectivity, were used in dose-effect experiments. Specifically, total amounts of $5 \times 10^{6}$ and $5 \times 10^{5}$ lactobacilli cells were tested.

\section{Nile Red Staining}

Lactobacillus crispatus BC5 was chosen as a model strain to study the molecular mechanisms underlining the interactions among lactobacilli, CT EBs and HeLa cells plasma membrane. HeLa cells were grown at $70-80 \%$ of confluence on glass coverslips, treated with $L$. crispatus BC5, S. agalactiae, E. faecalis, or B. subtilis cells $\left(5 \times 10^{7} \mathrm{CFU}\right)$ for $1 \mathrm{~h}$ at $37^{\circ} \mathrm{C}$ with $5 \% \mathrm{CO}_{2}$, and then washed three times with PBS. A stock solution of Nile Red (NR, 9-diethylamino-5H-benzo[alpha]phenoxazine-5one; Sigma-Aldrich, Milan, Italy) was prepared in DMSO at the concentration of $1 \mathrm{mM}$ and stored protected from light. $\mathrm{NR}$ staining was performed for $5 \mathrm{~min}$ at $37^{\circ} \mathrm{C}$ at a final dye concentration of $5 \mu \mathrm{M}$. Cells were then fixed with $3 \%$ paraformaldehyde for $10 \mathrm{~min}$, and repeatedly washed with $0.1 \mathrm{M}$ glycine/PBS and 1\% Bovine Serum Albumin (BSA)/PBS. Specimens were embedded in Mowiol and analyzed by a Nikon Coolscope II equipped with an Eclipse 90i microscope, using sequential laser excitations at $568 \mathrm{~nm}$ to reduce spectral bleedthrough artifacts. A Nikon Plan Apo $60 \times$ oil objective was used. Fluorescence intensity was quantified on at least eight randomly chosen microscopic fields by using Image J; results were expressed as average fluorescence (A.U.)/field \pm SD.

\section{Fluorescence Anisotropy Measurements}

The plasma membrane fluidity of HeLa cells was estimated by means of the fluorescence anisotropy of the hydrophobic probe TMA-DPH (1-4-trimethylammoniumphenil-6-phenil1,3,5-hexatriene; Thermo Fisher Scientific). HeLa cells (70-80\% confluence) were incubated with $L$. crispatus BC5, S. agalactiae, E. faecalis, or B. subtilis cells $\left(5 \times 10^{7} \mathrm{CFU}\right)$ for $1 \mathrm{~h}$ at $37^{\circ} \mathrm{C}$ with $5 \% \mathrm{CO}_{2}$, then washed three times with PBS and re-suspended at a final concentration of $3 \times 10^{5}$ cells $/ \mathrm{mL}$. The absorbance of the cell suspension was kept lower than 0.15 at the excitation wavelength of TMA-DPH. A few microliters of TMA-DPH stock solution were added to the cell suspension in order to obtain a final probe concentration of $1 \mu \mathrm{M}$. Fluorescence anisotropy measurements were performed by using a PTI QuantaMaster fluorimeter (Photon Technology International, North Edison, NJ, United States) equipped with a temperature-controlled cell holder and Polaroid HNP'B polarizers. Temperature was kept at $25^{\circ} \mathrm{C}$. Excitation and emission wavelengths were set at 360 and $430 \mathrm{~nm}$, respectively.

\section{Immunocytochemical Integrin Staining}

HeLa cells were grown on glass coverslips at $70-80 \%$ of confluence and treated with L. crispatus BC5, S. agalactiae, E. faecalis, or $B$. subtilis cells $\left(5 \times 10^{7} \mathrm{CFU}\right)$ for $1 \mathrm{~h}$ as described above, then washed three times with PBS and fixed in paraformaldehyde. Samples were stained with anti-human CD49e (i.e., anti-human $\alpha 5$ integrin subunit) primary antibody (1:500 in 1\% BSA/PBS, BioLegend, San Diego, CA, United States) overnight at $4^{\circ} \mathrm{C}$, followed by incubation with Alexa 568conjugated secondary antibody (1:1000 in 1\% BSA/PBS, Thermo 
Fisher Scientific) for $1 \mathrm{~h}$ at room temperature. As a specificity control for $\alpha 5$ integrin subunit signal, untreated Hela cells were also stained with IgG isotype (1:500 in 1\% BSA/PBS, SigmaAldrich), followed by Alexa 568-conjugated secondary antibody. Specimens were embedded in Mowiol and analyzed in confocal microscopy as described above. Fluorescence intensity was quantified on at least eight randomly chosen microscopic fields by using Image J; results were expressed as average fluorescence (A.U.)/field \pm SD.

\section{Exclusion Assay With Anti - CD49e Antibody}

In experiments exploring the role of the $\alpha 5$ integrin subunit in CT infection, HeLa cells grown on glass coverslips at $60 \%$ of confluence were first pre-exposed to control (IgG isotype) or anti-CD49e antibody (10 $\mu \mathrm{g} / \mathrm{mL})$ (BioLegend) for $1 \mathrm{~h}$ and then incubated with $5 \times 10^{3} \mathrm{CT}$ EBs for $48 \mathrm{~h}$ at $37^{\circ} \mathrm{C}$ and $5 \% \mathrm{CO}_{2}$. At the end of the incubation, samples were extensively washed in PBS, fixed in paraformaldehyde, permeabilized in ethanol and stained with the fluorescein-conjugated chlamydial membrane lipopolysaccharide antibody as described above. Specimens were embedded in Mowiol and analyzed in confocal microscopy as described above, using a Nikon Plan Apo $40 \times$ oil objective. Number of IFUs/field were counted by using Image J.

\section{$\alpha 5$ Integrin Subunit Silencing}

siRNA silencing was applied on HeLa cells after $48 \mathrm{~h}$ of adhesion, when cells were $60 \%$ confluent. The specific siRNA against ITGA5 gene expression and the scramble siRNA were transfected using lipofectamine according to the manufacturer's specifications (Thermo Fisher Scientific). Control siRNA or ITGA5 siRNA (10 nM) were used to treat cells in phenol red-, serum-, and antibiotic-free RPMI. After $48 \mathrm{~h}$ of siRNA treatment, cells were infected with $5 \times 10^{3} \mathrm{CT}$ EBs for additional $48 \mathrm{~h}$ at $37^{\circ} \mathrm{C}$ and $5 \% \mathrm{CO}_{2}$. Samples were then washed three times with PBS, fixed in paraformaldehyde, and permeabilized in ethanol. Cells were stained for chlamydial membrane lipopolysaccharide and analyzed by confocal microscopy as described above.

\section{Western Blot}

Control, siRNA, and scramble HeLa cells were washed twice with PBS and lysed for $1 \mathrm{~h}$ in lysis buffer (HEPES, $\mathrm{pH}$ $7.440 \mathrm{mM}$, glycerophosphate $60 \mathrm{mM}$, p-nitrophenylphosphate $20 \mathrm{mM}, \mathrm{Na}_{3} \mathrm{PO}_{4} 0.5 \mathrm{mM} \mathrm{NaCl} 250 \mathrm{mM}$, Triton X-100 1\%, PMSF $0.5 \mathrm{mM}$, and $10 \mathrm{mg} / \mathrm{mL}$ each of aprotinin, leupeptin, pepstatin and antipain, Sigma-Aldrich) at $0^{\circ} \mathrm{C}$. Cell lysates were centrifuged at $12,000 \times g$ for $20 \mathrm{~min}$. Supernatants were collected and protein concentration determined by using the Bio-Rad protein assay method (Bio-Rad, Hercules, CA, United States). The proteins were resolved on a $7.5 \%$ polyacrylamide gel and immunoblotted with a rabbit anti-human $\alpha 5$ integrin subunit (1:1000 in PBS Tween, Cell Signaling Technology, Danvers, MA, United States), or a rabbit anti- $\beta$-actin (1:2000 in PBS Tween, Sigma-Aldrich) antibodies. Detection of immunoreactive bands was performed by using a HRP-conjugated secondary antibody (1:20,000 in PBS Tween, GE Healthcare, Milan, Italy) followed by WESTAR
EtaC 2.0 (Cyanagen, Bologna, Italy). Densitometry analysis of immunoreactive bands was done by Fluor-S Max MultiImager (Bio-Rad). Relative quantification of $\alpha 5$ integrin subunit was performed by using $\beta$-actin signal as control.

\section{Statistical Analysis}

Infectivity data were analyzed by using $\mathrm{R}$ computational software ${ }^{1}$, applying the non-parametric one-tailed Wilcoxon rank test. Fluorescence intensities were analyzed by Prism software (GraphPad Software, La Jolla, CA, United States), applying the Student's $t$-test. Differences were deemed significant for $P$-values $\leq 0.01$.

\section{RESULTS}

\section{Interference of Lactobacilli With CT Infection}

In the present work we investigated the capability of vaginal lactobacilli to counteract the infection process of $C$. trachomatis in HeLa cells, chosen as an in vitro epithelial model of cervical infection. Specifically, we tested the inhibitory activity of 15 Lactobacillus strains, previously isolated from vaginal swabs of healthy premenopausal women (Parolin et al., 2015) through an exclusion assay. The effects of lactobacilli cells against C. trachomatis infection are reported in Figure $\mathbf{1}$ and the raw data are listed in Supplementary Table S1. All the Lactobacillus strains significantly reduced the chlamydial infection. The most active strains were L. crispatus BC1, BC3, BC4, BC5, BC6, L. gasseri $\mathrm{BC} 9, \mathrm{BC} 11, \mathrm{BC} 14$, and L. vaginalis $\mathrm{BC} 16$.

We excluded that the observed anti-CT activity was related to an acidic environment, since the $\mathrm{pH}$ values of the culture media were not modified by the addition and incubation with lactobacilli cells: in particular, $\mathrm{pH}$ of DMEM without lactobacilli ranged between 8.0 and 8.1 , whereas the $\mathrm{pH}$ values at 1 and $2 \mathrm{~h}$ post-incubation with lactobacilli ranged between 8.0 and 8.3.

In order to verify if the observed anti-Chlamydia activity was restricted to Lactobacillus strains, exclusion experiments were also performed with other Gram-positive microorganisms, namely $S$. agalactiae, E. faecalis, and $B$. subtilis. No reduction of Chlamydia infectivity was noticed for these control strains (Figure 1). In particular, as B. subtilis has similar shape and size of lactobacilli, we can suppose that the inhibition exerted by lactobacilli is not due to a physical barrier created by the large rod-shaped bacteria, but to a specific interaction with HeLa cells membrane.

\section{Dose-Response Effect of Lactobacilli on CT Infectivity}

We sought to investigate the effect of different doses of lactobacilli cells on the level of inhibition of CT infection. For this purpose, we selected three strains among the most active ones (L. crispatus BC4, L. crispatus BC5, and L. gasseri BC14) and we evaluated the inhibitory effects of two dilutions, corresponding to the doses

\footnotetext{
${ }^{1}$ www.R-project.org
} 


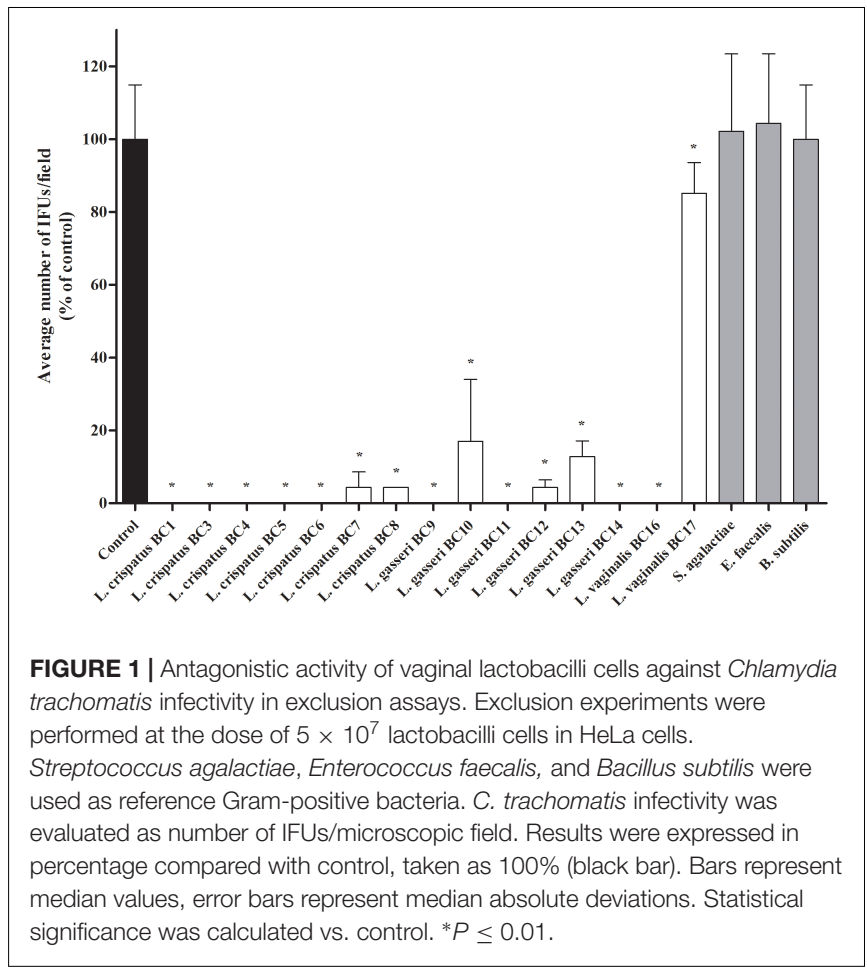

of $5 \times 10^{6}$ and $5 \times 10^{5}$ cells (Figure 2). All Lactobacillus strains significantly reduced Chlamydia infectivity, confirming the strong antagonistic effect of Lactobacillus cells toward the infectious process of Chlamydia. Despite the antagonist activity was maintained even at lower doses, the level of inhibition was clearly dose dependent for all the lactobacilli.

\section{L. crispatus BC5 Modifies HeLa Cells Plasma Membrane}

We focused our investigation on L. crispatus BC5, chosen as model strain. We thus wondered if $1 \mathrm{~h}$-treatment of Hela cells with $L$. crispatus BC5 cells prior to the infection with CT would be able to induce modifications at the HeLa plasma membrane level, in terms of lipid organization, fluidity, and modulation of protein exposure. HeLa cells were stained with the lipid dye NR and analyzed by confocal microscopy (Figure 3A). The interaction of $L$. crispatus BC5 with HeLa caused a decrease in NR emission fluorescence (Fluorescence intensity A.U. control: $37093 \pm 8347$, BC5: $16616 \pm 6257, P=0.001$ ), indicating a reduced exposure of polar membrane lipids. Steady-state fluorescence anisotropy of TMA-DPH was used to evaluate the possible modifications of the physico-chemical characteristics of HeLa plasma membrane upon interaction with L. crispatus BC5. Under our experimental conditions, in the absence of L. crispatus BC5, an anisotropy value of $0.266 \pm 0.002$ was measured. The treatment with Lactobacillus cells induced a significant decrease in TMA-DPH anisotropy (0.257 \pm 0.001 ; $P=0.006)$.

It is known that numerous pathogens, including C. trachomatis, exploit integrins expressed on target cell

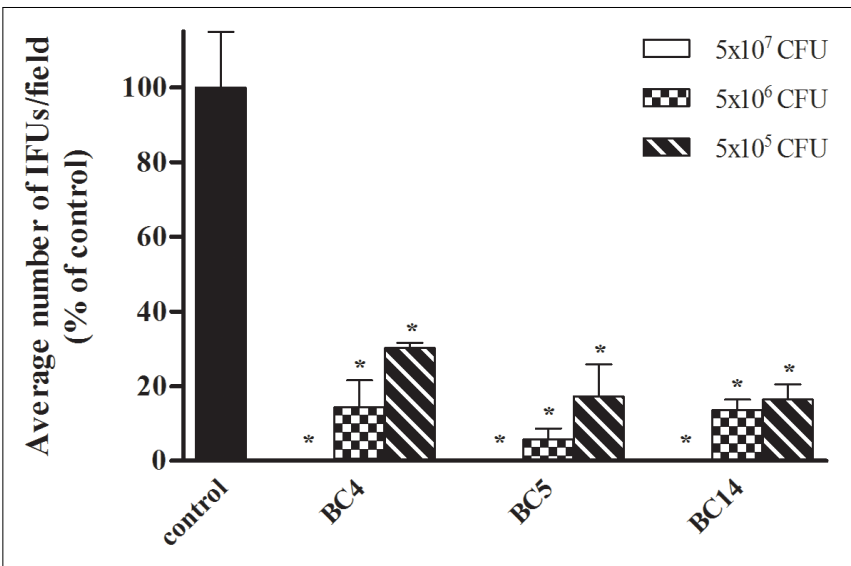

FIGURE 2 | Dose-response effect of Lactobacillus cells on C. trachomatis infectivity in HeLa cells. Exclusion experiments were performed at different doses $\left(5 \times 10^{7}, 5 \times 10^{6}\right.$, and $5 \times 10^{5}$ cells) for L. crispatus BC4, L. crispatus $B C 5$, and L. gasseri BC14 strains. C. trachomatis infectivity was evaluated as number of IFUs/microscopic field. Results were expressed in percentage compared with control, taken as 100\% (black bars). Bars represent median values, error bars represent median absolute deviations. Statistical significance was calculated vs. control. ${ }^{*} P \leq 0.01$.

plasma membrane in order to mediate adhesion and cell penetration. For this reason, we investigated whether L. crispatus BC5 pre-treatment could alter integrin exposure on $\mathrm{HeLa}$ plasma membrane, by immunostaining of CD49e and confocal microscopy analysis (Figure 3B). The pre-incubation of $\mathrm{HeLa}$ cells with $L$. crispatus BC5 significantly reduced the exposure of $\alpha 5$ integrin subunit on the plasma membrane (Fluorescence intensity A.U. control: $41449 \pm 4765$, BC5: $27453 \pm 3056$, $P=0.001)$. Specificity of $\alpha 5$ integrin subunit staining was verified by using IgG isotype followed by fluorescent secondary antibody (Fluorescence intensity A.U. IgG: $3878 \pm 337$ ).

In order to verify if the modification of membrane properties are restricted to L. crispatus BC5, the same experiments were also performed with other Gram-positive microorganisms. HeLa cells were thus incubated with S. agalactiae, E. faecalis, and B. subtilis. The interaction of $S$. agalactiae and E. faecalis with HeLa cells caused a strong decrease in NR emission fluorescence (Figure 3A) (Fluorescence intensity A.U. S. agalactiae: $16484 \pm 5048$, E. faecalis: $12911 \pm 3413, P=0.001$ vs. control, for both comparisons), but these effects on membrane lipid organization were not associated with changes in the membrane exposure of $\alpha 5$ integrin subunit (Figure 3B) (Fluorescence intensity A.U. S. agalactiae: $40549 \pm 4986$, $P=0.319$; E. faecalis: $40059 \pm 1333, P=0.214)$. B. subtilis treatment did not affect HeLa plasma membrane lipid organization (Figure 3A) (NR Fluorescence intensity A.U. $42424 \pm 7803, P=0.338)$ nor $\alpha 5$ integrin subunit exposure (Figure 3B) (Fluorescence intensity A.U. $42655 \pm 3073$, $P=0.142$ ). The measurement of anisotropy in Hela cells incubated with S. agalactiae, E. faecalis, and B. subtilis was not performed because these control microorganisms incorporated the TMA-DPH probe. 

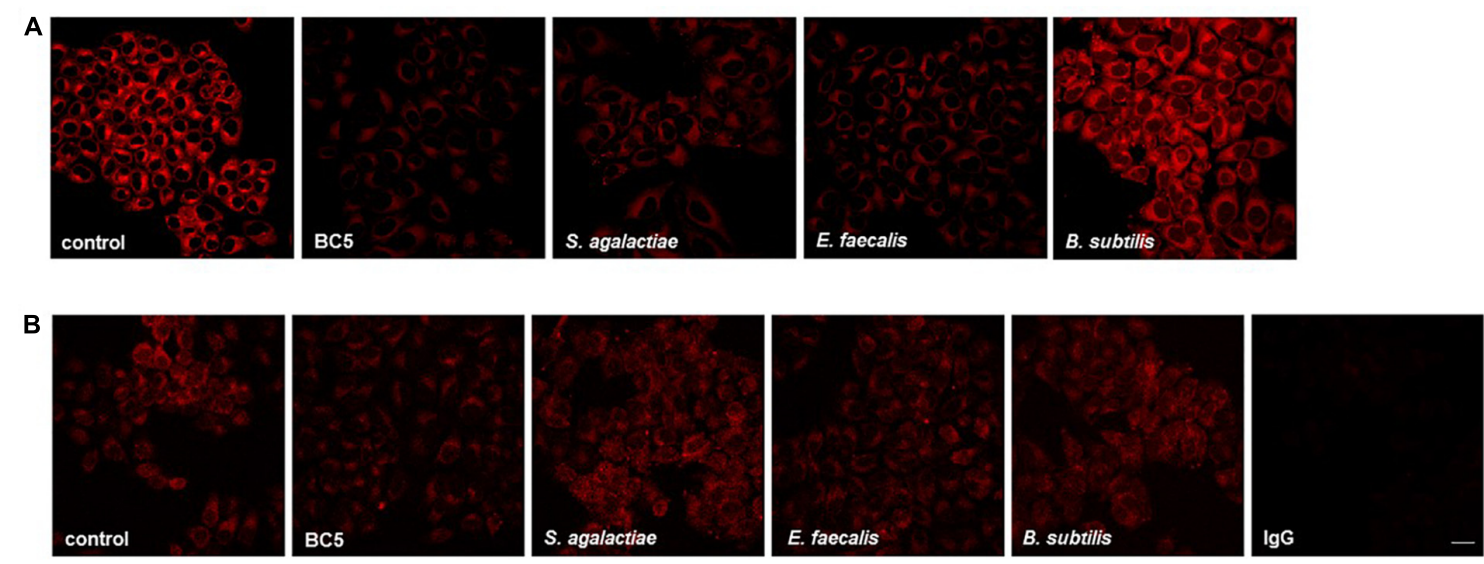

FIGURE 3 | Membrane lipid organization and $\alpha 5$ integrin exposure on HeLa cells incubated with microorganisms. (A) HeLa cells were incubated with $L$. crispatus BC5, S. agalactiae, E. faecalis, or B. subtilis for $1 \mathrm{~h}$ and then stained with NR. (B) HeLa cells were incubated with L. crispatus BC5, S. agalactiae, E. faecalis, $B$. subtilis for $1 \mathrm{~h}$ and then stained for $\alpha 5$ integrin subunit. IgG represents specificity staining control. Representative micrographs are shown. Experiments were repeated at least three times with similar results. Bar: $20 \mu \mathrm{m}$.

\section{Blocking or Silencing of $\alpha 5$ Integrin Subunit Prevent CT Infection}

To examine the role of $\alpha 5 \beta 1$ in CT infectious process in HeLa cells, CT infectivity was evaluated after pre-incubation of $\mathrm{HeLa}$ cells with a function-blocking anti- $\alpha 5$ integrin subunit antibody or control IgG. Internalization of CT EBs by HeLa cells was reduced by approximately $60 \%$ in the presence of the anti-CD49e antibody $(P<0.01)$, while the pre-incubation with isotype IgG did not interfere with $C$. trachomatis infection (Figures 4A,B).

To further confirm the involvement of $\alpha 5$ integrin in CT infectious process in HeLa cells, we used a specific ITGA5 siRNA to knockdown endogenous $\alpha 5$ integrin subunit expression. Validated siRNAs were used for the analysis: a specific antiITGA5 and a correspondent negative siRNA were chosen (scramble). A silencing time course was assessed by quantification of $\alpha 5$ integrin subunit protein $48-120 \mathrm{~h}$ after silencing with siRNA $10 \mathrm{nM}$. Total protein lysates were resolved in SDS-PAGE and $\alpha 5$ integrin subunit protein was identified by a specific antibody in western blot. By normalization on the amount of $\beta$-actin, quantification of $\alpha 5$ integrin subunit in the specific siRNA sample was compared with the relative scramble sample (Figures 4C,D). Since $\alpha 5$ integrin subunit expression was already greatly reduced after $48 \mathrm{~h}$ of siRNA treatment, this time point was chosen as optimal. Following silencing, cells were infected with CT EBs. Transfection of cells with $\alpha 5$ integrin-specific siRNA reduced $\mathrm{CT}$ infection by $64 \%$. In contrast, transfection of cells with non-specific control siRNA did not affect CT infection (Figures 4E,F).

\section{DISCUSSION}

The vaginal microbiota of healthy pre-menopausal women is dominated by different species of Lactobacillus. They play a fundamental role in the maintenance of microbial homeostasis of the female genital tract, preventing the overgrowth of endogenous opportunistic microorganisms and impeding the colonization of exogenous pathogens (Aroutcheva et al., 2001; Borges et al., 2014). Women with a depletion of vaginal lactobacilli are more likely to be infected by urogenital and sexually transmitted pathogens, such as chlamydia, gonorrhea, and HIV (Gallo et al., 2012; Alcaide et al., 2015).

Although many studies have focused on the mechanisms displayed by lactobacilli in counteracting several urogenital pathogens (Osset et al., 2001; Verdenelli et al., 2014; Breshears et al., 2015; Parolin et al., 2015), up to now only a few data are available about the interaction between lactobacilli and CT. Gong et al. (2014) demonstrated that lactobacilli could inactivate chlamydiae primarily through maintaining an acidic environment in the vaginal lumen by the production of lactic, formic, and acetic acids. Nardini et al. (2016) confirmed the capability of lactobacilli secreted metabolites to strongly inhibit CT EBs infectivity and identified L. crispatus as the species with the best anti-Chlamydia profile, due to the high production of lactate and the marked consumption of glucose. Moreover, in the same work it has been demonstrated that lactobacilli cells can directly inactivate CT EBs, probably by means of rapid and dynamic modifications of EBs membrane, although to a lesser extent than supernatants. The peculiarity of these previous studies is that the interactions between lactobacilli and CT EBs were simulated in 'external killing' experiments, where target epithelial cells were not present. Indeed, in such assays, CT EBs were incubated with lactobacilli supernatants/cells prior to contact with the epithelial cells and residual EBs infectivity was subsequently evaluated. Considering that chlamydiae are obligate intracellular organisms, it is essential to investigate the contemporary interplays of CT, vaginal lactobacilli and host epithelium. For this reason, here we evaluated the capacity of Lactobacillus cells to interfere with CT infectivity, placing both microorganisms in contact with Hela cells, which represent the cervical epithelium. 

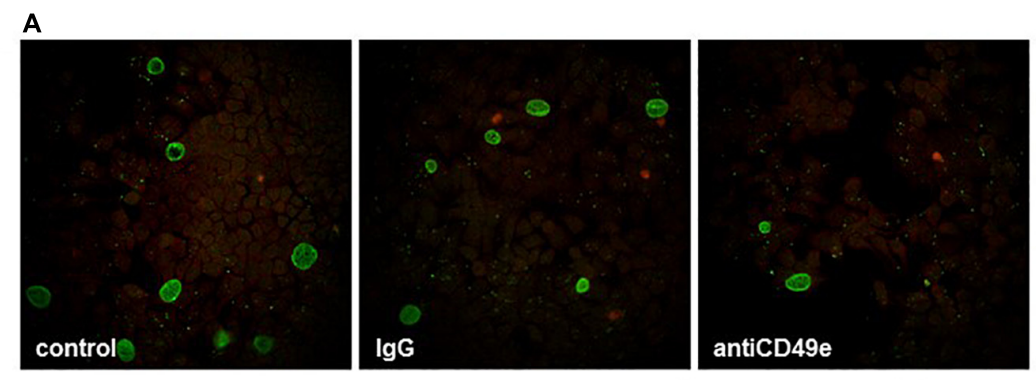

C

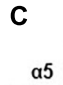

$140 \mathrm{kD}$

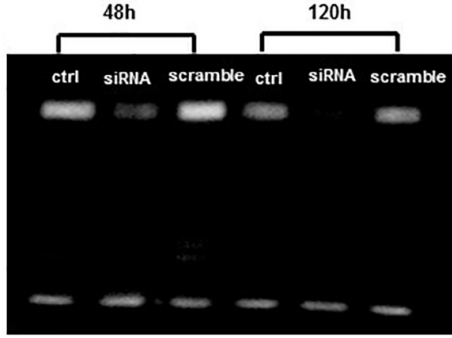

E
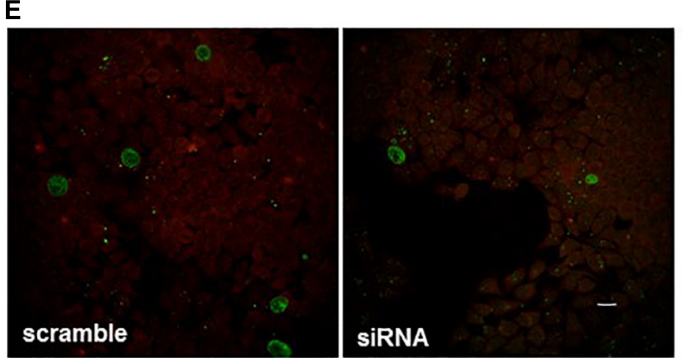

B

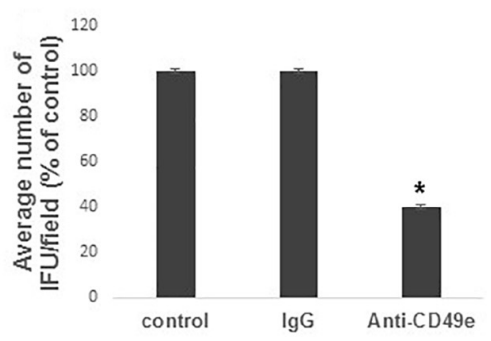

D

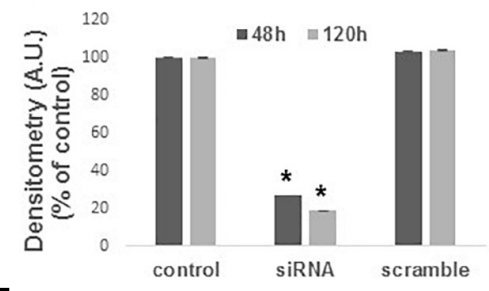

$\mathbf{F}$

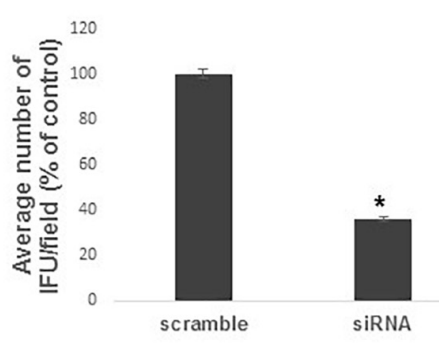

FIGURE 4 | Inhibition of $C$. trachomatis infection by $\alpha 5$ integrin subunit blocking or ITGA5 gene silencing. HeLa cells were treated or not with an anti-CD49e antibody or control lgG for $1 \mathrm{~h}$, then infected with CT EBs for $48 \mathrm{~h}$. (A,B) Specimens were stained for chlamydial membrane lipopolysaccharide antigen. Representative micrographs are shown. C. trachomatis infectivity was evaluated as number of IFUs/microscopic field. Results were expressed in percentage compared with control, taken as $100 \%$. Bars represent median values, error bars represent median absolute deviations. Statistical significance was calculated vs. control. ${ }^{*} P \leq 0.01$. (C,D) Western blotting of $\alpha 5$ integrin subunit expression in control, ITGA5 siRNA and scramble Hela cells, evaluated at 48 and $120 \mathrm{~h}$ post-siRNA. Quantification of $\alpha 5$ integrin subunit was normalized on $\beta$-actin. Bars represent mean values based on three independent experiments, error bars represent standard deviations. (E,F) HeLa cells treated with siRNA or scramble were infected with CT EBs for $48 \mathrm{~h}$, and then stained for chlamydial membrane lipopolysaccharide antigen. Bar: $20 \mu \mathrm{m}$. Results were expressed in percentage compared with scramble, taken as $100 \%$. Bars represent median values, error bars represent median absolute deviations. Statistical significance was calculated vs. control. $* P \leq 0.01$

First we found a strong antagonistic activity of Lactobacillus cells against the infectious process of CT by means of an exclusion strategy, suggesting that the physiological colonization of the female genital tract by lactobacilli is crucial to prevent the intracellular penetration of the pathogen. Such antagonistic activity is specific for Lactobacillus strains, as other Gram-positive microorganisms, i.e., S. agalactiae, E. faecalis and B. subtilis, failed to interfere with CT infectivity process. In this context, the use of probiotic formulations able to create a cervico-vaginal niche rich in 'health-promoting' Lactobacillus species could be an intriguing approach for the prevention of chlamydial infections and other STIs.

Notably, the present data, integrated with the literature, suggest that vaginal lactobacilli play an important role in countering chlamydial infection exerting concerted and synergetic effects at different stages of the infection process. The metabolites secreted by lactobacilli are able to reduce CT EBs viability during the early infection steps before their internalization into epithelial cells, as previously reported (Nardini et al., 2016). Regarding Lactobacillus cells, here we have demonstrated that they act with great effectiveness in preventing the interaction of Chlamydia with the eukaryotic cells, by means of exclusion. Although a dose-dependent effect was noticed, a significant antagonistic activity was maintained for all the lactobacilli tested even at lower doses. The fact that low amounts of lactobacilli still exert a significant anti-Chlamydia activity prompted us to assume that the prevention of CT infection could be ascribed to modifications in the membrane of epithelial cells, that become less susceptible to CT penetration. Moreover, as previously described for Candida spp. (Parolin et al., 2015), we excluded that the most adhesive lactobacilli strains were the most active against CT, strengthening the hypothesis that other mechanisms, besides a steric encumbrance and a saturation of the adhesion sites, should be taken into account.

In the perspective to understand the biochemical bases of the protection exerted by Lactobacillus, we further studied 
the modifications induced by $L$. crispatus BC5 on the host cell membrane, in terms of lipid organization, fluidity, and modulation of protein exposure. We chose L. crispatus BC5 as model strain for the following reasons: (i) high inhibitory activity of BC5 toward CT, demonstrated in the present work; (ii) suitability of BC5 to be included in pharmaceutical preparations aimed at the delivery of probiotics to the vaginal mucosa, demonstrated in our previous study (Vitali et al., 2016). Particularly, the interest in a potential application of $L$. crispatus BC5 in pharmaceutical formulations has prompted us to deepen in this strain the knowledge of the molecular mechanisms underlying its antimicrobial action.

It has been previously demonstrated that other vaginal lactobacilli determine strain-specific modifications in the physical properties of HeLa cell plasma membrane. These effects were related to the protective role of lactobacilli against the adhesion of Candida albicans on the epithelial cells (Calonghi et al., 2017). Some Lactobacillus strains (e.g., L. crispatus BC1 and L. gasseri BC9) caused a reduced exposure of polar lipids in HeLa plasma membrane with an increase of membrane fluidity. Modification in membrane fluidity could affect receptor binding function by modifying the receptor availability and its rotational and lateral motions. In addition, alterations in lipid fluidity could lead to changes in the tertiary and quaternary structure of the receptor compromising the bacterial ligands recognition (Salyer et al., 1990). Other Lactobacillus strains (e.g., L. gasseri BC11 and L. vaginalis BC15) did not influence HeLa plasma membrane fluidity but they changed $\alpha 5 \beta 1$ integrin exposure. Particularly, these strains modified $\alpha 5 \beta 1$ integrin organization, leading to the formation of characteristic membrane protein clusters. The consequence of these membrane modifications could be a deviation from receptors native conformation with the resultant inability to correctly recognize and interact with bacterial ligands.

In this work we demonstrated that the incubation of HeLa cells with $L$. crispatus BC5 cells induces important modifications at the level of the epithelial cell plasma membrane, by altering both lipid composition and protein exposure. Upon interaction with lactobacilli, HeLa plasma membrane became more fluid, and reduced the exposure of polar lipids and $\alpha 5 \beta 1$ integrin subunits. Notably, when a specific blocking antibody masked $\alpha 5$ integrin subunits or the corresponding gene expression was silenced, CT infection was strongly reduced, supporting the hypothesis that $\alpha 5$ subunit is crucial for CT infection in HeLa cells. This finding, in accordance with Stallmann and Hegemann (2016), confirms the role of integrins as key mediators of the pathogenic process of C. trachomatis, and indicates the involvement of

\section{REFERENCES}

Abbai, N. S., Reddy, T., and Ramjee, G. (2015). Prevalent bacterial vaginosis infection - a risk factor for incident sexually transmitted infections in women in Durban, South Africa. Int. J. STD AIDS 27, 1283-1288. doi: 10.1177/ 0956462415616038

Alcaide, M. L., Chisembele, M., Malupande, E., Arheart, K., Fischl, M., and Jones, D. L. (2015). A cross-sectional study of bacterial vaginosis, intravaginal practices the $\alpha 5 \beta 1$ dimer in the CT-host cell interaction. However, the adhesion and invasion of CT into epithelial cells rely on other host factors in addition to integrins, such as mannose receptors, ephrin receptor A2 and fibroblast growth factor receptors (FGFR) (Elwell et al., 2016; Lujan et al., 2018). Therefore, it is not surprising that the blocking of $\alpha 5$ integrin subunits or the ITGA5 gene expression silencing are responsible of only a partial, but substantial, reduction of CT infectivity, rather than a complete abolishment of it.

\section{CONCLUSION}

Our results allowed us to delineate a mechanism of protection by $L$. crispatus BC5 directly linked to membrane properties modifications in epithelial cells, in particular to the reduction of $\alpha 5 \beta 1$ integrin exposure. We are aware that other complementary mechanisms, in addition to the modulation of integrin exposure, could contribute to the ability of lactobacilli to reduce CT infectivity into epithelial cells. Further perspectives will include the study of the modulation of other CT receptors on cervical cells after the interaction with different strains of vaginal lactobacilli.

\section{AUTHOR CONTRIBUTIONS}

$\mathrm{BV}, \mathrm{AM}$, and $\mathrm{NC}$ conceived and designed the study, supervised the experimental work, and data analysis. CP, GF, CF, BG, and MS performed the experiments and the statistical analysis. CP, $\mathrm{BV}$, and CF drafted the manuscript. All authors read, reviewed and approved the final manuscript, and contributed in data interpretation.

\section{FUNDING}

This work was supported by 'Fondazione del Monte di Bologna e Ravenna' and by national funds (MIUR, Ministry of Education, University and Research, Italy).

\section{SUPPLEMENTARY MATERIAL}

The Supplementary Material for this article can be found online at: https://www.frontiersin.org/articles/10.3389/fmicb. 2018.02630/full\#supplementary-material

and HIV genital shedding; implications for HIV transmission and women's health. BMJ Open 5:e009036. doi: 10.1136/bmjopen-2015-009036

Aroutcheva, A., Gariti, D., Simon, M., Shott, S., Faro, J., Simoes, J. A., et al. (2001). Defense factors of vaginal lactobacilli. Am. J. Obstet. Gynecol. 185, 375-379. doi: $10.1067 / \mathrm{mob} .2001 .115867$

Barrons, R., and Tassone, D. (2008). Use of Lactobacillus probiotics for bacterial genitourinary infections in women: a review. Clin. Ther. 30, 453-468. doi: 10.1016/j.clinthera.2008.03.013 
Bolton, M., Van Der Straten, A., and Cohen, C. R. (2008). Probiotics: potential to prevent HIV and sexually transmitted infections in women. Sex. Transm. Dis. 35, 214-225. doi: 10.1097/OLQ.0b013e31815b017a

Borges, S., Silva, J., and Teixeira, P. (2014). The role of lactobacilli and probiotics in maintaining vaginal health. Arch. Gynecol. Obstet. 289, 479-489. doi: 10.1007/ s00404-013-3064-3069

Breshears, L. M., Edwards, V. L., Ravel, J., and Peterson, M. L. (2015). Lactobacillus crispatus inhibits growth of Gardnerella vaginalis and Neisseria gonorrhoeae on a porcine vaginal mucosa model. BMC Microbiol. 15:276. doi: 10.1186/s12866015-060

Calonghi, N., Parolin, C., Sartor, G., Verardi, L., Giordani, B., Frisco, G., et al. (2017). Interaction of vaginal Lactobacillus strains with HeLa cells plasma membrane. Benef. Microbes 8, 625-633. doi: 10.3920/BM2016.0212

ECDC (2015). Sexually Transmitted Infections in Europe 2013. Stockholm: European Centre for Disease Prevention and Control.

Elwell, C., Mirrashidi, K., and Engel, J. (2016). Chlamydia cell biology and pathogenesis. Nat. Rev. Microbiol. 14, 385-400. doi: 10.1038/nrmicro.2016.30

Foschi, C., Salvo, M., Cevenini, R., Parolin, C., Vitali, B., and Marangoni, A. (2017). Vaginal lactobacilli reduce Neisseria gonorrhoeae viability through multiple strategies: an in vitro study. Front. Cell. Infect. Microbiol. 7:502. doi: 10.3389/ fcimb.2017.00502

Gallo, M. F., Macaluso, M., Warner, L., Fleenor, M. E., Hook, E. W. IIIrd, Brill, I., et al. (2012). Bacterial vaginosis, gonorrhea, and chlamydial infection among women attending a sexually transmitted disease clinic: a longitudinal analysis of possible causal links. Ann. Epidemiol. 22, 213-220. doi: 10.1016/j.annepidem. 2011.11.005

Gong, Z., Luna, Y., Yu, P., and Fan, H. (2014). Lactobacilli inactivate Chlamydia trachomatis through lactic acid but not H2O2. PLoS One 9:e107758. doi: 10. 1371/journal.pone. 0107758

Gupta, K., Stapleton, A. E., Hooton, T. M., Roberts, P. L., Fennell, C. L., and Stamm, W. E. (1998). Inverse association of H2O2-producing lactobacilli and vaginal Escherichia coli colonization in women with recurrent urinary tract infections. J. Infect. Dis. 178, 446-450. doi: 10.1086/515635

Hauck, C. R., Borisova, M., and Muenzner, P. (2012). Exploitation of integrin function by pathogenic microbes. Curr. Opin. Cell Biol. 24, 637-644. doi: 10.1016/j.ceb.2012.07.004

Hoffmann, C., Ohlsen, K., and Hauck, C. R. (2011). Integrin-mediated uptake of fibronectin-binding bacteria. Eur. J. Cell Biol. 90, 891-896. doi: 10.1016/j.ejcb. 2011.03.001

Hynes, R. O. (2002). Integrins: bidirectional, allosteric signaling machines. Cell 110, 673-687. doi: 10.1016/S0092-8674(02)00971-976

Kaewsrichan, J., Peeyananjarassri, K., and Kongprasertkit, J. (2006). Selection and identification of anaerobic lactobacilli producing inhibitory compounds against vaginal pathogens. FEMS Immunol. Med. Microbiol. 48, 75-83. doi: 10.1111/j. 1574-695X.2006.00124.x

Lujan, A. L., Croci, D. O., Gambarte Tudela, J. A., Losinno, A. D., Cagnoni, A. J., Mariño, K. V., et al. (2018). Glycosylation-dependent galectin-receptor interactions promote Chlamydia trachomatis infection. Proc. Natl. Acad. Sci. U.S.A. 115, E6000-E6009. doi: 10.1073/pnas.1802188115

Marangoni, A., Fiorino, E., Gilardi, F., Aldini, R., Scotti, E., Nardini, P., et al. (2015). Chlamydia pneumoniae acute liver infection affects hepatic cholesterol and triglyceride metabolism in mice. Atherosclerosis 241, 471-479. doi: 10.1016/ j.atherosclerosis.2015.05.023

Martin, H. L., Richardson, B. A., Nyange, P. M., Lavreys, L., Hillier, S. L., Chohan, B., et al. (1999). Vaginal lactobacilli, microbial flora, and risk of human immunodeficiency virus type 1 and sexually transmitted disease acquisition. J. Infect. Dis. 180, 1863-1868. doi: 10.1086/315127

Mastromarino, P., Di Pietro, M., Schiavoni, G., Nardis, C., Gentile, M., and Sessa, R. (2014). Effects of vaginal lactobacilli in Chlamydia trachomatis infection. Int. J. Med. Microbiol. 304, 654-661. doi: 10.1016/j.ijmm.2014.04.006

Menon, S., Timms, P., Allan, J. A., Alexander, K., Rombauts, L., Horner, P., et al. (2015). Human and pathogen factors associated with Chlamydia trachomatisrelated infertility in women. Clin. Microbiol. Rev. 28, 969-985. doi: 10.1128/ CMR.00035-15
Moulder, J. W. (1991). Interaction of chlamydiae and host cells in vitro. Microbiol. Rev. 55, 143-190.

Ñahui Palomino, R. A., Zicari, S., Vanpouille, C., Vitali, B., and Margolis, L. (2017). Vaginal Lactobacillus inhibits HIV-1 replication in human tissues ex vivo. Front. Microbiol. 8:906. doi: 10.3389/fmicb.2017.00906

Nardini, P., Nahui Palomino, R. A., Parolin, C., Laghi, L., Foschi, C., Cevenini, R., et al. (2016). Lactobacillus crispatus inhibits the infectivity of Chlamydia trachomatis elementary bodies, in vitro study. Sci. Rep. 6:29024. doi: 10.1038/ srep29024

Osset, J., Bartolomé, R. M., García, E., and Andreu, A. (2001). Assessment of the capacity of Lactobacillus to inhibit the growth of uropathogens and block their adhesion to vaginal epithelial cells. J. Infect. Dis. 183, 485-491. doi: 10.1086/ 318070

Parolin, C., Marangoni, A., Laghi, L., Foschi, C., Ñahui Palomino, R. A., Calonghi, N., et al. (2015). Isolation of vaginal lactobacilli and characterization of anti-candida activity. PLoS One 10:e0131220. doi: 10.1371/journal.pone. 0131220

Price, M. J., Ades, A. E., De Angelis, D., Welton, N. J., MacLeod, J., Soldan, K., et al. (2013). Risk of pelvic inflammatory disease following Chlamydia trachomatis infection: analysis of prospective studies with a multistate model. Am. J. Epidemiol. 178, 484-492. doi: 10.1093/aje/kws583

Salyer, J. L., Bohnsack, J. F., Knape, W. A., Shigeoka, A. O., Ashwood, E. R., and Hill, H. R. (1990). Mechanisms of tumor necrosis factor-alpha alteration of PMN adhesion and migration. Am. J. Pathol. 136, 831-841.

Spurbeck, R. R., and Arvidson, C. G. (2008). Inhibition of Neisseria gonorrhoeae epithelial cell interactions by vaginal Lactobacillus species. Infect. Immun. 76, 3124-3130. doi: 10.1128/IAI.00101-108

Spurbeck, R. R., and Arvidson, C. G. (2011). Lactobacilli at the front line of defense against vaginally acquired infections. Future Microbiol. 6, 567-582. doi: $10.2217 / \mathrm{fmb} .11 .36$

Stallmann, S., and Hegemann, J. H. (2016). The Chlamydia trachomatis Ctad1 invasin exploits the human integrin $\beta 1$ receptor for host cell entry. Cell. Microbiol. 18, 761-775. doi: 10.1111/cmi.12549

Taha, T. E., Hoover, D. R., Dallabetta, G. A., Kumwenda, N. I., Mtimavalye, L. A. R., Yang, L. P., et al. (1998). Bacterial vaginosis and disturbances of vaginal flora: association with increased acquisition of HIV. AIDS 12, 1699-1706. doi: 10.1097/00002030-199813000-199813019

Verdenelli, M. C., Coman, M. M., Cecchini, C., Silvi, S., Orpianesi, C., and Cresci, A. (2014). Evaluation of antipathogenic activity and adherence properties of human Lactobacillus strains for vaginal formulations. J. Appl. Microbiol. 116, 1297-1307. doi: 10.1111/jam.12459

Vitali, B., Abruzzo, A., Parolin, C., Nahui Palomino, R. A., Dalena, F., Bigucci, F., et al. (2016). Association of Lactobacillus crispatus with fructo-oligosaccharides and ascorbic acid in hydroxypropyl methylcellulose vaginal insert. Carbohydr. Polym. 136, 1161-1169. doi: 10.1016/j.carbpol.2015.10.035

Wiesenfeld, H. C., Hillier, S. L., Krohn, M. A., Landers, D. V., and Sweet, R. L. (2003). Bacterial vaginosis is a strong predictor of Neisseria gonorrhoeae and Chlamydia trachomatis infection. Clin. Infect. Dis. 36, 663-668. doi: 10.1086/ 367658

Younes, J. A., Lievens, E., Hummelen, R., van der Westen, R., Reid, G., and Petrova, M. I. (2018). Women and their microbes: the unexpected friendship. Trends Microbiol. 26, 16-32. doi: 10.1016/j.tim.2017.07.008

Conflict of Interest Statement: The authors declare that the research was conducted in the absence of any commercial or financial relationships that could be construed as a potential conflict of interest.

Copyright (C) 2018 Parolin, Frisco, Foschi, Giordani, Salvo, Vitali, Marangoni and Calonghi. This is an open-access article distributed under the terms of the Creative Commons Attribution License (CC BY). The use, distribution or reproduction in other forums is permitted, provided the original author(s) and the copyright owner(s) are credited and that the original publication in this journal is cited, in accordance with accepted academic practice. No use, distribution or reproduction is permitted which does not comply with these terms. 